\title{
Acute Brachial Artery Occlusion following Blunt Trauma
}

\author{
Kwon-Jae Park \\ Department of Trauma Surgery, Won-Kwang University Hospital, Iksan, Korea
}

When symptoms of a threatened a limb is observed in a patient with acute arterial occlusion, prompt revascularization should be performed to salvage the limb and reduce complications. However, this care may be delayed in various clinical situations. Here, we report a clinical experience of a brachial artery occlusion in the situation mentioned above.

(Trauma Image Proced 2017(2):87-89)

Key Words: Brachial artery; Wound and injuries; Ischemia; Surgery; Upper extremity.

\section{CASE}

A 43-year-old male patient presented to the emergency room with left arm pain. He fell to the ground from $2 \mathrm{~m}$ while working and his left arm struck a heavy metallic material. He had widespread bruising on the arm and a 7-8 $\mathrm{cm}$ laceration wound near the left axilla (Fig. 1). Physical examination revealed that the left radial and ulnar pulses were decreased compared to the right-sided pulses but were obviously palpable and audible using Doppler Ultrasound. The skin was not pale or not. The motor grade of the left arm was slightly decreased. There was numbness evident in the $3 \mathrm{rd}, 4^{\text {th }}$, and 5th fingers. A vascular Upper-Extremity CT angiography was performed and showed the absence of flow in the mid-brachial artery, which preserved flow of radial and ulnar arteries (Fig. 2). An operation was not immediately performed due to the patient not meeting the threatened limb criteria (e.g., palpable pulses, relatively warm skin). Finally, the patient was transferred to the operation room 12 hours following the accident.

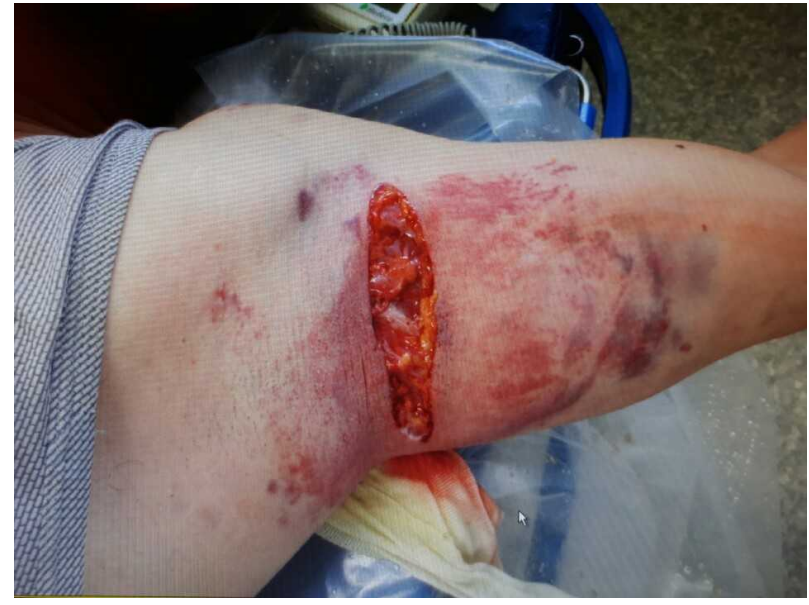

Fig. 1. A 7-8 cm long laceration wound and a contusion on the left arm were found.

Operation revealed that the brachial artery was injured in the middle portion. The injured part of the artery showed no pulse and contained thrombotic material that totally occluded the artery (Fig. 3). The portion was resected and interposed with sections from the great saphenous vein. There was no obvious injury to the

Received: September 6, 2017 Revised: October 13, 2017 Accepted: October 25, 2017

Correspondence to: Kwon-Jae Park, Department of Trauma Surgery, Won-Kwang University Hospital, 895, Muwang-ro, Iksan-si, Jeollabuk-do, Korea

Tel: 82-63-859-2532, Fax: 82-63-859-2532, E-mail: juvabien@hanmail.net

Copyright (c) 2017 Korean Association for Research, Procedures and Education on Trauma. All rights reserved.

@This is an open-access article distributed under the terms of the Creative Commons Attribution Non-Commercial License (http://creativecommons.org/ licenses/by-nc/4.0) which permits unrestricted noncommercial use, distribution, and reproduction in any medium, provided the original work is properly cited 


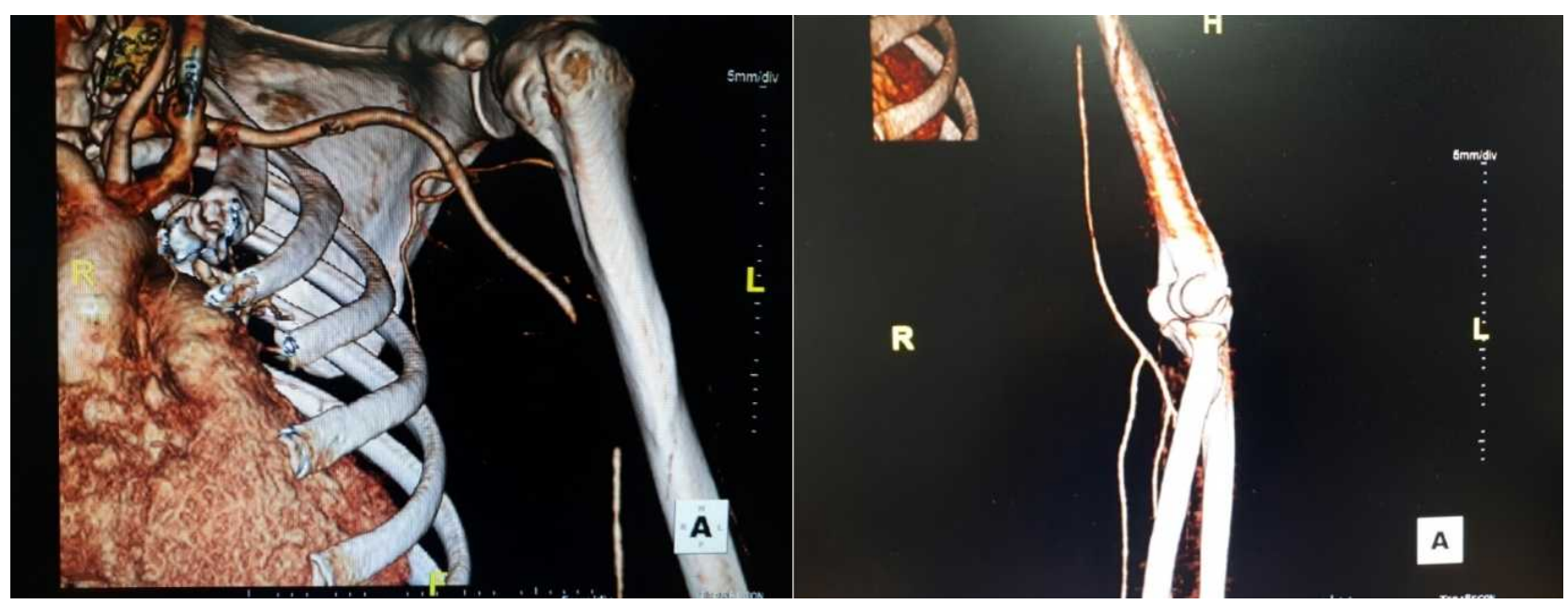

Fig. 2. Vascular Upper-Extremity CT angiography revealed no flow on the mid-brachial artery. The flow of radial \& ulnar arteries was preserved.

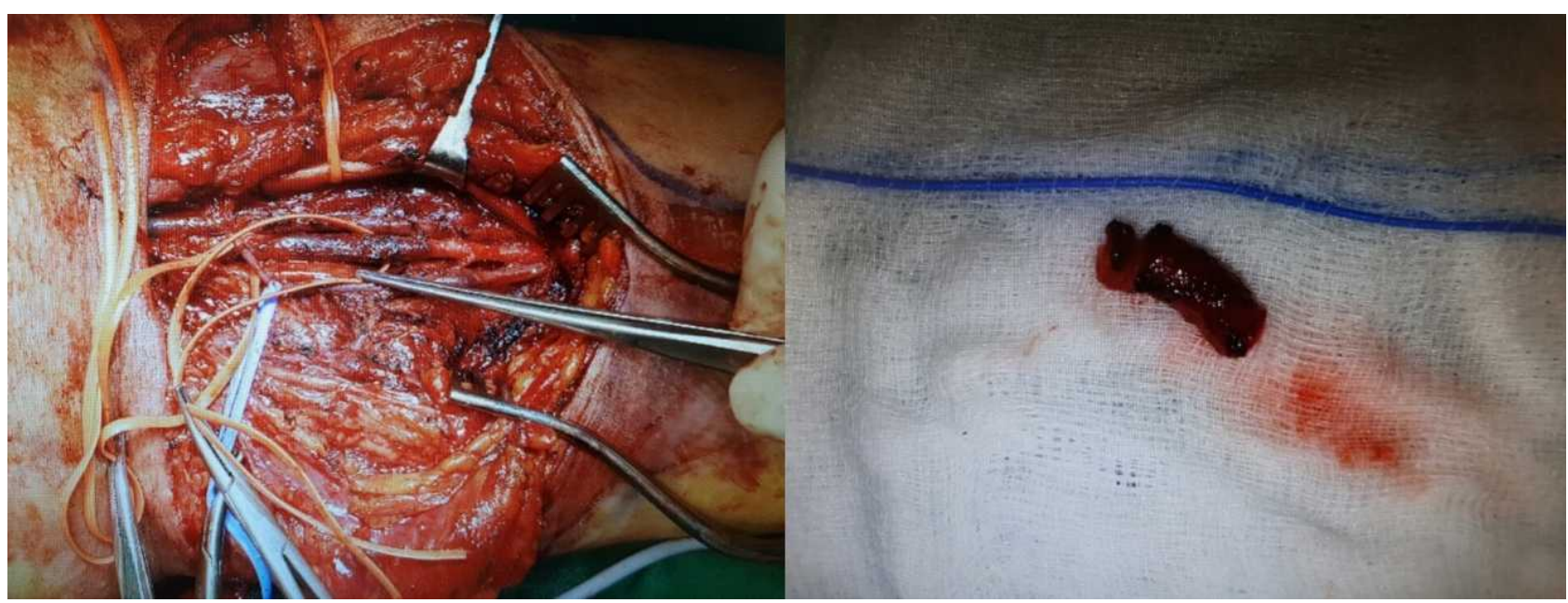

Fig. 3. The mid-portion of the brachial artery was severely injured presenting with a blackish discoloration and no pulsation, and it was filled with thrombotic material that totally occluded the arterial lumen.

median nerve. Following the procedure, the strength of arterial pulses on the forearm was restored to the that of the right side. On postoperative day two, there were no problems observed in the patient. However, left arm swelling began by postoperative 3 day and became aggravated thereafter. The radial pulse also decreased due to edema. The patient complained of a bursting out sense of left arm preserving the pulses in the forearm. Fortunately, swelling didn`t increase. Also, other severe complications like compartment syndrome didn`t develop. Swelling of the arm eventually improved, and the pulse was restored. At postoperative 11 days, a vascular Upper-Extremity CT angiography showed the recovery of the flow in the artery, however, swelling of the forearm persisted (Fig. 4). By postoperative day 25, the patient was discharged without complications and has been followed up by an outpatient department.

\section{DISCUSSION}

Clinical manifestation of an acute arterial occlusion includes coldness, pain, pallor, pulseless, paresthesia and 


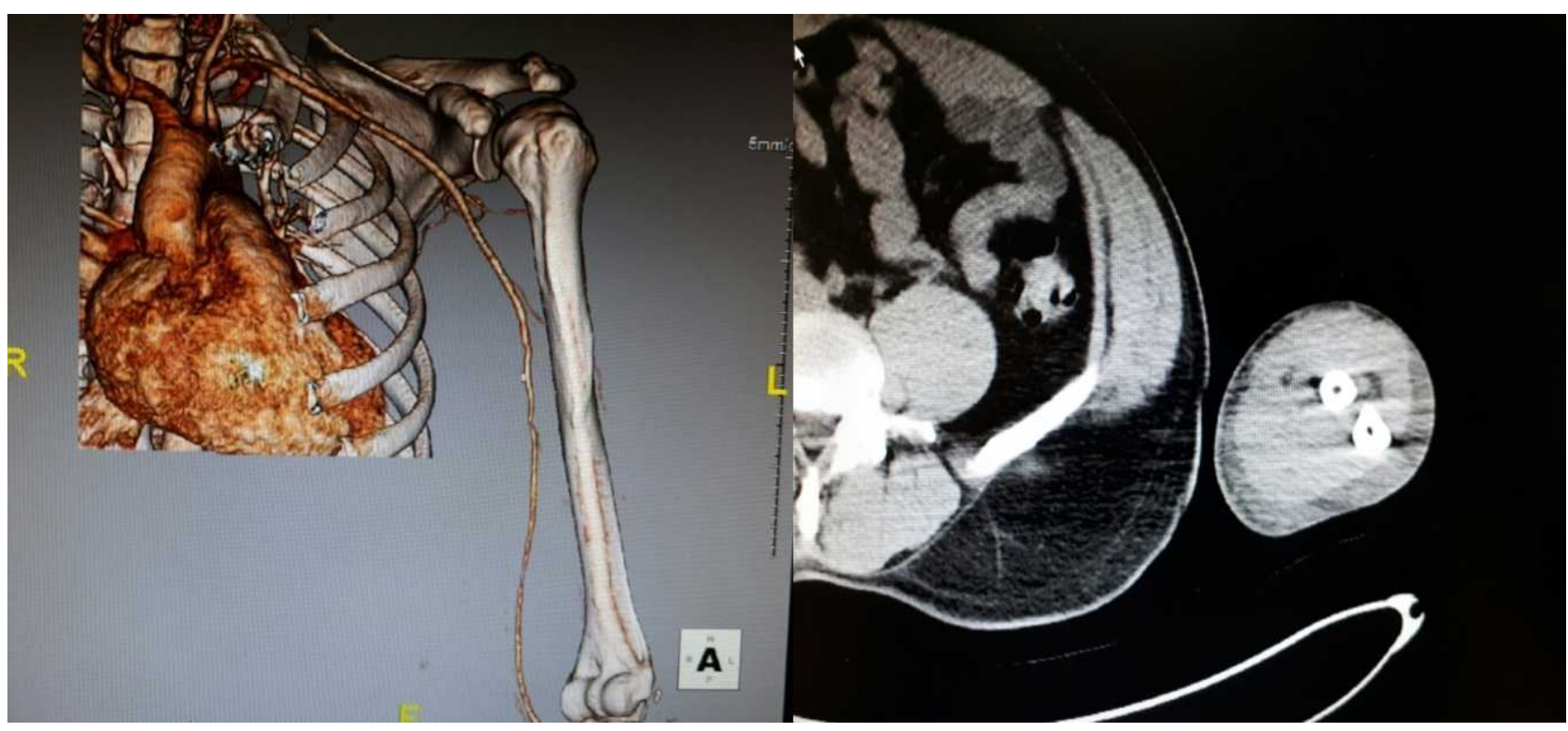

Fig. 4. Postoperative vascular angio CT showed the recovery of flow in the brachial artery. Edema in the forearm was still present.

paralysis. If some of them are identified in a patient's limb, we should suspect highly of acute limb ischemia and prepare an emergent intervention or operation to salvage the limb and avoid serious postoperative complications. However, when it comes to acute brachial occlusion, acute signs of acute limb ischemia might be obscured due to naturally abundant upper extremity collaterals $(1,2)$. So delayed diagnosis and operation may be possible. In general, no flow of the artery is identified distal to the obstruction in vascular angio-CT. However, distal arteries like the radial and ulnar artery were found in this case. To minimize ischemic-reperfusion injury following the operation, it is important to limit the preoperative ischemic period. Beyond the golden period of 6 to 8 hours of ischemia, reperfusion injury such as compartment syndrome and rhabdomyolysis may develop and threaten the patient's life (3). Therefore, prompt diagnosis and operation should be employed even if there is no obvious evidence of an acute brachial occlusion.

\section{Conflict of Interest Statement}

No potential conflict of interest relevant to this article was reported.

\section{REFERENCES}

1. Levin PM, Rich NM, Hutton JE Jr. Collaternal circulation in arterial injuries. Arch Surg 1971;102:392-9.

2. McCroskey BL, Moore EE, Pearce WH, Moore FA, Cota $\mathrm{R}$, Sawyer JD. Traumatic injuries of the brachial artery. Am J Surg. 1988;156:553 - 555.

3. Zellweger R, Hess F, Nicol A, Omoshoro-Jones J, Kahn D, Navsaria P. An analysis of 124 surgically managed brachial artery injuries. Am J Surg. 2003;188:240 - 245. 